\title{
Multiple Layers in Black Gloss as Evidence of Multiple Firings
}

\author{
Ilaria Cianchetta ${ }^{2}$, Karen Trentelman ${ }^{2}$, Marc Walton $^{3}$, Apurva Mehta ${ }^{4}$, Elisa Maupas ${ }^{2 *}$, Brendan Foran ${ }^{1}$ \\ ${ }^{1}$ The Aerospace Corporation, 2350 E. El Segundo Blvd., El Segundo, CA 90245-4691 \\ 2 The Getty Conservation Institute, 1200 Getty Center Dr., Los Angeles, CA 90049 \\ ${ }^{3}$ Art Institute of Chicago, Northwestern University, Chicago, Il \\ ${ }^{4}$ Stanford Synchrotron Radiation Laboratory, 2575 Sandhill Rd., Menlo Park, CA 94025 \\ * Centre for Archaeological Sciences, K.U.Leuven, Leuven, Belgium
}

In this work we present a compositional and morphological study of black gloss ceramic slips from Athenian black figure pottery (dated 430 B.C.). By evaluating the relationship between composition and porosity the firing protocol may be inferred, helping further our understanding of the development and evolution of ancient ceramic technologies.

The iconic red and black figure vessels, produced in Athens from the 6th through the 4th centuries B.C., represent the pinnacle of ancient ceramic craftsmanship. The ancient Greeks painted the ceramics with refined Fe-rich clay that, when fired, created deep black decorative glossy surfaces. The refinement process likely involved isolation of very fine grain clays through levigation; dispersing the clay into large vats of water, allowing the coarse grains to settle to the bottom, leaving fine grains suspended in solution for subsequent collection. The established model ${ }^{[1]}$ describes the firing process as a single firing in which the kiln environment was subjected to a three stage cycle of oxidation-reduction-oxidation. When fired at high temperature under reducing conditions the slips sintered and turned from red (hematite, $\mathrm{Fe}_{2} \mathrm{O}_{3}$ ) to black (magnetite, $\mathrm{Fe}_{3} \mathrm{O}_{4}$, hercynite $\mathrm{FeAl}_{2} \mathrm{O}_{4}$ ). During the last step, oxygen was unable to diffuse into the more vitrified slips, keeping them black, whereas the iron minerals in the porous body ceramic were reoxidized to $\mathrm{Fe}_{2} \mathrm{O}_{3}$, producing a contrasting red color. As previously reported $^{[2]}$ the production of this pottery was likely more complex than formerly thought, with vessels subjected to two, or possibly more, firings in the kiln, with applications of slip between each firing.

X-ray fluorescence (XRF) elemental mapping of a number of ancient shards from the collection of the $\mathrm{J}$. Paul Getty Museum revealed elevated levels of $\mathrm{Zn}$ in the gloss, often with different components on a single vessel containing different amounts of $\mathrm{Zn}$. The measured levels were inconsistent with the natural occurrence of this element in soil, suggesting it may have been introduced during the levigation process. XRF analysis of a cross section sample consisting of a black gloss relief line painted over background gloss (both painted over the red reserve) was conducted to determine the composition of the two different gloss applications throughout their entire thickness (see Figure 1, upper). The presence of two chemically different superimposed layers of black gloss is evident: the top layer contains significantly higher $\mathrm{Zn}$ than the lower layer. X-ray absorption near edge spectroscopy (XANES) of the Fe K-edge for the two layers showed that the upper layer, higher in $\mathrm{Zn}$, is composed primarily of hercynite, while the lower layer is more magnetite-like (see Figure 1, lower). A focused ion beam (FIB) lift-out cross-section extending slightly into the underlying ceramic shard body allowed a TEM morphological analysis of both gloss layers to be conducted (see Figure 2). The porosity was observed to decrease from bottom to top, with a much denser and vitrified texture corresponding to the high-Zn hercynite-like layer at the surface. The presence of two layers with different morphology, different mineralogy and different chemical composition is presented as evidence of a complex firing sequence following the application of each decorative motif. 


\section{References}

[1] J.V. Noble, The Techniques of Painted Attic Pottery (Watson-Guptill, New York) 1965

[2] M. Walton, et al. Journal of American Ceramic Society 96, 7, (2013), p. 2031

[3] This material is based upon work supported by the National Science Foundation under Grant No. 1041808. The authors also gratefully acknowledge Jeffrey Maish and David Saunders of the J. Paul Getty Museum for their contributions to this work.
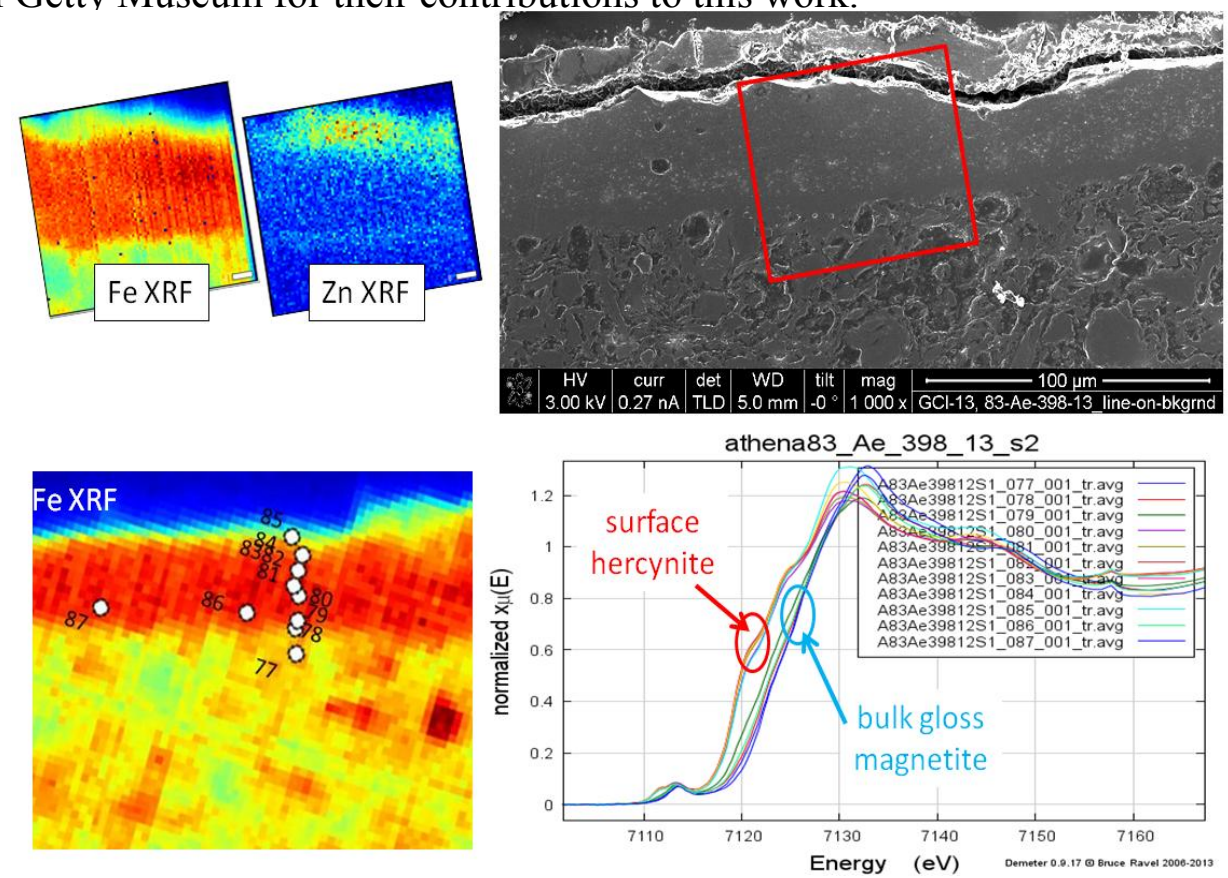

Figure 1. Top: Fe and Zn XRF maps and SEM image of black gloss cross section. Bottom: Fe XRF map of cross section with spot analysis through thickness and XANES spectra of corresponding spots.

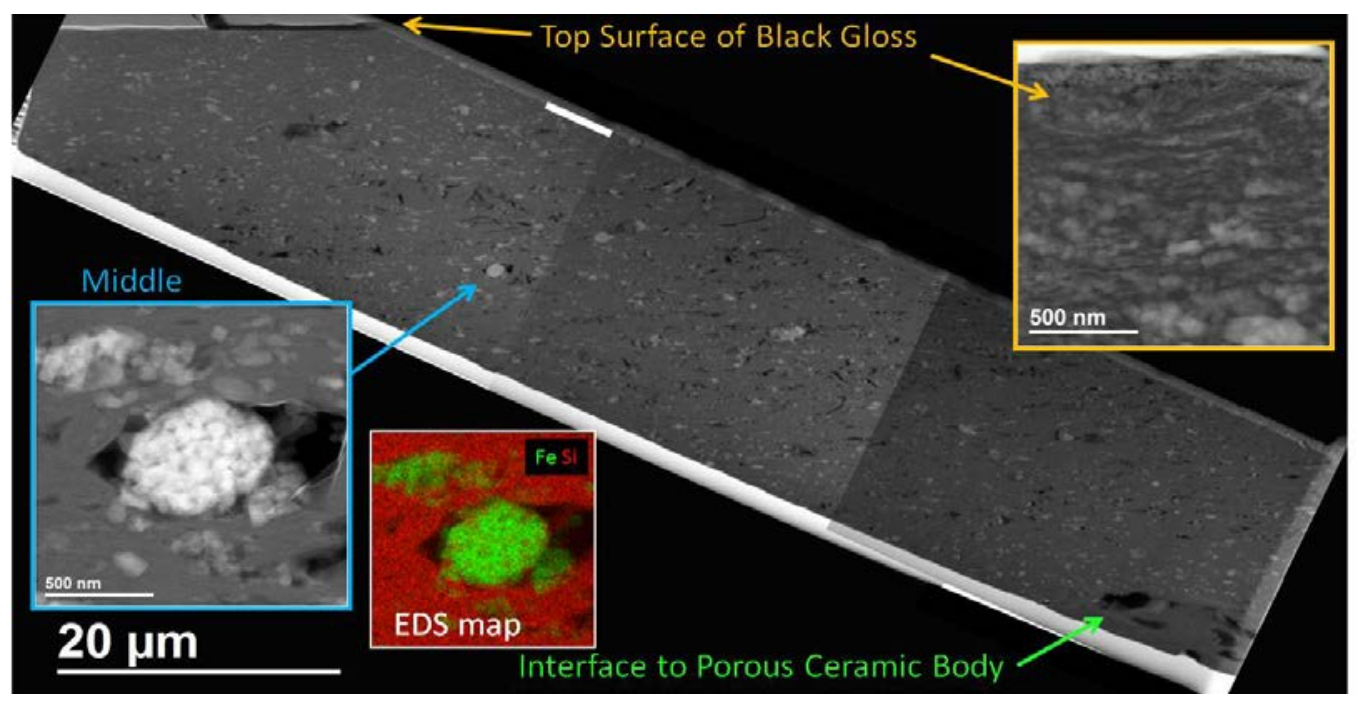

Figure 2. High angle annular dark field scanning transmission electron microscopy images showing focused ion beam prepared cross-section through full thickness (tilted) of black gloss for sample with inset images showing detail near the top surface and middle portions of the black gloss. 